Check for updates

Cite this: Nanoscale Adv., 2019, 1, 4174

Aqeel Al-Ani, ${ }^{\star a b}$ Josiah J. C. Haslam, ${ }^{a}$ Natalie E. Mordvinova, ${ }^{c}$ Oleg I. Lebedev, ${ }^{c}$ Aurélie Vicente, ${ }^{d}$ Christian Fernandez ${ }^{d}$ and Vladimir Zholobenko ${ }^{* a}$

DOI: $10.1039 / \mathrm{c} 9 \mathrm{na} 90049 \mathrm{~g}$

rsc.li/nanoscale-advances

\section{Correction: Synthesis of nanostructured catalysts by surfactant-templating of large-pore zeolites}

Correction for 'Synthesis of nanostructured catalysts by surfactant-templating of large-pore zeolites' by Aqeel Al-Ani et al., Nanoscale Adv., 2019, 1, 2029-2039.

The following amendments have been suggested:

\title{
Introduction
}

The surfactant-templated mesostructuring approach, introduced in ref. 21 and 25, allows for a more precise control of the intracrystalline mesoporosity introduced while retaining the main properties of the zeolites, including their microporosity, catalytic activity, and hydrothermal stability. For these reasons, surfactant-templated zeolites have been successfully commercialised. $^{22,36}$

\section{Experimental section}

The preparation of mesostructured faujasites followed the general procedure introduced in ref. 25.

\section{Results and discussion}

In agreement with previous reports, ${ }^{18,25,44}$ our results demonstrate that TIPB conversion increases for all zeolites following their mesostructuring treatment.

\section{Acknowledgments}

The authors would like to thank Professor Javier Garcia-Martinez and Dr Eric Li for the useful information provided regarding the synthesis of surfactant-templated zeolites.

\section{References}

The reference numbers cited here correspond to those in the original paper.

The Royal Society of Chemistry apologises for these errors and any consequent inconvenience to authors and readers. 